\title{
Real-time control of inertial focusing in microfluidics using dielectrophoresis (DEP)
}

\author{
Jun Zhang, ${ }^{a}$ Sheng Yan, ${ }^{a}$ Gursel Alici, ${ }^{a}$ Nam-Trung Nguyen, ${ }^{* b}$ Dino Di Carlo ${ }^{* c d}$ and Weihua Li $*^{a}$ \\ Received (in $X X X, X X X$ ) Xth $X X X X X X X X X 20 X X$, Accepted $X$ th $X X X X X X X X X 20 X X$ \\ DOI: $10.1039 / b 000000 x$
}

In this paper we propose a novel hybrid microfluidic device which, for the first time, integrates and fully couples both dielectrophoresis (DEP) and inertial focusing. A DEP force is coupled with inertial lift force to adjust particle equilibrium positions in the vertical direction in real time. The focusing pattern and position of the particles along the horizontal plane can be adjusted concurrently by adjusting their vertical position with DEP forces. The magnitude of secondary flow drag increases dramatically when particles are levitated towards the centre of the channel in the vertical direction. The paper investigates the mechanism of this tuneable DEP-inertial microfluidic device analytically and experimentally. The proposed hybrid device possesses the advantages of both DEP and inertial microfluidic devices, working in a high-throughput manner as well as having precise controllability in real-time. This DEP-inertial microfluidic device is potentially a versatile and robust platform for feedback-controlled manipulation and separation of particles and cells.

\section{Introduction}

Microfluidic technology has achieved significant progress over the last decade. This fascinating technology holds broad application areas including sample preparation ${ }^{1}$, clinical diagnosis ${ }^{2}$, waste water treatment ${ }^{3}$, drug screening ${ }^{4}$ and material synthesis, ${ }^{5}$ etc. Manipulation of particles such as biological cells is an essential capability in microfluidics to analyse biochemical processes at the single-cell level or to separate cells according to their unique bio-physical signatures.

Various techniques have already been proposed and developed to manipulate particles in microfluidics. According to the source of the manipulating force, they can be categorised as active and passive techniques. Active techniques such as dielectrophoresis (DEP) ${ }^{6}$, magnetophoresis (MP) ${ }^{7}$ and acoustophoresis (AP) ${ }^{8}$ rely on an external force field, whereas passive techniques depend entirely on the channel geometry or intrinsic hydrodynamic forces, such as pinched flow fractionation (PFF) ${ }^{9}$, deterministic lateral displacement (DLD) ${ }^{10}$ and inertial microfluidics ${ }^{11}$. An active technique generally allows for a more precise control of target particles, while being very flexible for a wide range of biological particles and tuneable in real-time. However, the flow speed is always limited because particles must be exposed to the outer force field for sufficient duration to achieve effective functionality, which reduces their throughput. In contrast, a passive microfluidic device is always very robust and has a high throughput. However, the fixed geometry and design of passive devices limit their manipulative capability, making them only effective for a specific range of particle properties. That means, separating particles out of the previous design range would need a completely new device design. Ideally then, a combination of highthroughput passive techniques with precisely tuneable active ones would result in a more powerful and versatile platform.

Attempts to combine active and passive techniques in one microfluidic device are rarely reported in the past. ${ }^{12-15}$ Ozkumur et al. ${ }^{13}, 14$ reported a hybrid microfluidic device which combined deterministic lateral displacement (DLD), inertial focusing and magnetophoresis (MP) to isolate rare circulating tumour cells (CTCs) in an antigen-dependent and independent way. In this device, after a sample of raw blood is introduced into the chip, the blood cells first encounter an array of circular pillars with a specific gap, where DLD size-based hydrodynamic force filtrate smaller red blood cells (RBCs) from white blood cells (WBCs) and CTCs with much larger size. Subsequently, the mixture of WBCs and CTCs flows into an asymmetric serpentine channel where they are promptly focused along a single path by the inertial effects within a serpentine channel. At the end of the serpentine channel, a strong magnetic field is applied to deflect those cells that are magnetically labelled. The CTCs can then be collected separately. Benefiting from the initial debulking of RBCs by DLD and its serpentine inertial focusing section, this device allows for a throughput as high as $10^{7}$ cells per second. Another impressive work was reported by Moon et al. ${ }^{15}$ who successfully separated human breast cancer cells (MCF-7) from spiked blood sample by a combination of multi-orifice flow fractionation (MOFF) and dielectrophoresis (DEP) techniques. The inertial separation by MOFF takes advantage of the high-throughput filtration of blood cells, and the serially connected DEP separator works as a precise postprocessor to further enhance the efficiency and purity of the separation process. The paper claimed that $99.24 \%$ of RBCs and $94.23 \%$ of WBCs were removed, with a 162-fold increase in MCF-7 cells. In both works, the separators were actually connected in series, and no 
coupling between the separators exists.

To broaden the scope and capability of microfluidic technology further, we explored the possibility of combining DEP and inertial focusing in a fully-coupled manner, and proposed a new concept which is called DEP-inertial microfluidics. Rather than choosing a horizontal DEP force to directly deflect particles, a vertical DEP force was used to levitate particle equilibrium positions vertically to the centre of the channel. Due to the no-slip boundary, the fluid velocity at the centre of the channel is at its maximum, which imposes a much stronger secondary flow drag onto the particles. This means the inertial focusing pattern and position of the particles along the horizontal plane can even be tuned. The mechanism of this tuneable DEP-inertial microfluidic device was discussed, and the effects of the DEP force on each inertial focusing pattern were investigated. Our proposed hybrid device has the advantages of both DEP and inertial microfluidic devices, working in a high-throughput manner as well as with precise controllability. The concept reported here is expected to provide a versatile and robust platform for feedback-controlled manipulation and separation of particles and cells.

\section{Materials and Methods}

\section{A. Design and fabrication of the DEP-inertial hybrid device}

The serpentine channel used in our experiments consisted of 15 symmetric zigzag periods. The width of the micro-channel was $200 \mu \mathrm{m}$. The length and width of each U-turn were both $700 \mu \mathrm{m}$. The height of the channel was uniformly $40 \mu \mathrm{m}$. The polydimethylsiloxane (PDMS) slab embedded with the micro-channel was fabricated by standard photolithography and soft replica techniques. ${ }^{16}$ On the glass slide, a 50-nm Ti/150-nm Pt electrode layer was patterned using the standard lift-off process. ${ }^{17}$ The spacing and width of the interdigitated electrodes (IDEs) were both $20 \mu \mathrm{m}$. The IDEs were $13.5 \mathrm{~mm}$ long, which left one zigzag period of micro-channel uncovered by IDEs to allow for the clear observation of particle trajectory. The PDMS slab and glass slide were bonded with the help of oxygen plasma after being carefully aligned. Figure 1 shows a schematic image of the DEP-inertial microfluidic device.

\section{B. Particle preparation}

Internally dyed fluorescent polystyrene particles $(10 \mu \mathrm{m}$ in diameter, Thermo Fisher Scientific) were suspended in deionized (DI) water with $0.025 \% w / w$. Tween 20 (SIGMA-ALDRICH Product No. P9416) with $0.1 \% w / v$ was added to prevent the particles from aggregating. Bulk conductivity and permittivity of the polystyrene beads are $2.4 \times 10^{-4} \mathrm{~S} / \mathrm{m}$ and $2.6 \varepsilon_{o}$, respectively. The conductivity and permittivity of DI water are $1.5 \times 10^{-4} \mathrm{~S} / \mathrm{m}$ and $78 \varepsilon_{o}$, respectively. ${ }^{18}$

\section{Experimental setup and methods}

The microfluidic device was placed on an inverted microscope (CKX41, Olympus, Japan), illuminated by a mercury arc lamp. A particle suspension was delivered into the micro-channel by a syringe pump (Legato 100, KD Scientific). Fluorescent images were observed and captured by a CCD camera (Rolera Bolt, Q-imaging, Australia), and then post-processed and analysed using Q-Capture Pro 7 (Qimaging, Australia) software. The exposure time for each frame was consistent at $100 \mathrm{~ms}$. A sinusoidal signal with a frequency of $1 \mathrm{MHz}$ was generated by a waveform generator (33250A, Agilent, USA) and amplified by an RF power amplifier (TIA-1000-1R8-2, MiniCircuits, USA). The applied frequency in all experiments was set to $1 \mathrm{MHz}$. Copper wires with one end soldered onto the IDEs pads were connected to the power amplifier to activate the electrodes.

\section{Mechanism}

\section{A. Inertial migration}

The inertial focusing of particles in a straight channel is driven by the counteraction of two inertial effects: the shear gradient lift force $F_{L S}$, and the wall lift force $F_{L W}$. The shear gradient lift force $F_{L S}$ depends on the curvature of the fluid velocity profile and its interaction with a finite size particle, while the wall lift force $F_{L W}$ is a result of a disturbance of the flow field around the suspending particles and its reflection off the wall. The equilibrium positions result from the balance between these two inertial lift forces. The net inertial lift force $F_{L}$ can be expressed as follows when the particle size is small compared to the channel size: ${ }^{11,19}$

$$
\begin{gathered}
F_{L}=\frac{\rho_{f} U_{m}^{2} a^{4}}{D_{h}^{2}} f_{L}\left(R_{C}, z\right) \\
R_{C}=\frac{\rho_{f} U_{m} D_{h}}{\mu_{f}}
\end{gathered}
$$

where $\rho_{f}, U_{m}$ and $\mu_{f}$ are fluid density, maximum velocity, and dynamic viscosity, respectively; $a$ is particle diameter, and $D_{h}$ is hydraulic diameter of channel. $f_{L}\left(R_{C}, z\right)$ is the lift coefficient of the net inertial lift force, which is a function of position of the particles within cross section of channel $z$ and channel Reynolds number $R_{C} .{ }^{11}$ The situation of $f_{L}=0$ corresponds to the dynamic stable and unstable equilibrium positions for particles.

The introduction of channel curvature in the downstream direction introduces additional inertial forces from the fluid (secondary flow drag) and the particles (centrifugal force), which will modify and assist the inertial focusing progress. ${ }^{20}$ Normally the particle centrifugal 
force is negligible unless the particle has quite different density compared to the fluid. Subsequently, the focusing pattern (e.g. position and number of dynamic equilibrium positions) is always determined by the balance between secondary flow drag and inertial lift force. When secondary flow is too weak to alter inertial focusing, the focusing pattern is still determined by the inertial lift force, and particles are normally ordered on the two sides of a channel. ${ }^{21}$ If the secondary flow is strong enough, it can modify particles as a single stream in a curved channel from top view. ${ }^{20,22,23}$

\section{B. The n-DEP force in interdigitated electrodes (IDEs)}

Dielectrophoretic (DEP) forces occur on particles/cells when a non-uniform electrical field interacts with field-induced electrical polarisation. A particle immersed in an alternating electrical field is subject to a time-averaged DEP force determined by the frequencydependent polarisability of the particle relative to the medium, given by: ${ }^{24}$

$$
F_{D E P}=2 \pi \varepsilon_{m} r^{3} \operatorname{Re}[K(\omega)] \nabla\left|E_{r m s}\right|^{2}
$$

where $\varepsilon_{m}$ is the permittivity of the medium, $r$ represents the particle radius, $\left|E_{r m s}\right|$ is the root-mean-squared value of the applied electrical field, and $K(\omega)$ refers to the Clausius-Mossotti (CM) factor. Re indicates the real part of the factor, and $K(\omega)$ depends on the relative permittivity of the particle and the suspending medium, as well as the frequency of the external electric field.

$$
K(\omega)=\left(\varepsilon_{p}^{*}-\varepsilon_{m}^{*}\right) /\left(\varepsilon_{p}^{*}+2 \varepsilon_{m}^{*}\right)
$$

where $\varepsilon^{*}=\varepsilon-\mathrm{i} \sigma / \omega$ is the complex permittivity, $\sigma$ is the electrical conductivity, and $\omega$ is the frequency of the electric field. The subscripts $p$ and $m$ denote the particle and suspending medium, respectively.

When the dielectric constant of a particle is larger than the medium, $\operatorname{Re}[K(\omega)]>0$, the DEP is positive, and particles move towards the location with the highest electric field gradient. But when the dielectric constant of a particle is smaller than the medium, $\operatorname{Re}[K(\omega)]<0$, the DEP force is negative and particles move to locations with the lowest electric field gradient.

In the present work, an IDEs array was patterned onto the bottom of the micro-channel to generate a negative DEP (n-DEP) on the particles and push them upwards, Figure 2(a). Since the DEP force in the horizontal direction is relatively small and negligible, we focused on the vertical DEP force $\left(F_{D E P z}\right)$ and its counteraction with the inertial lift force along the vertical plane. Without a specific notice, the DEP force in the whole manuscript indicates the DEP force component in the vertical $(z)$ direction. Near the surfaces of the electrodes $(z \leq 10 \mu \mathrm{m})$, the magnitude of DEP force oscillates along the longitudinal $(x)$ direction. The peak points are on the edges of the electrodes, while the trough points are at the centre of the electrodes and their gaps. Moreover, the closer the particle to the electrode covered surface, the higher is the magnitude of the oscillation, Figure 2(b). Meanwhile, when particles move away from the surface of the electrodes, the magnitude of the DEP force decreases sharply as well as the magnitude of oscillation, Figure 2(b and c). However, in the region far from the surface of the electrodes $(z \geq 10 \mu \mathrm{m})$, the DEP force remains almost constant in the longitudinal direction. When fluid flows rapidly through this microchannel patterned with IDEs, e.g. $R_{C} \sim 100$ (or $U \sim 1 \mathrm{~m} / \mathrm{s}$ ), the particles suspended in the medium will follow the streamlines at a very high speed. The net effect on particles of the fluctuating DEP force depends on its average magnitude.

\section{Coupling of n-DEP force and inertial lift force along vertical direction}

If particles are close to the walls of the channel in the vertical direction $z$, a wall lift force $F_{L W}$, generated by the wall-induced disturbance on the flow field around the particles, pushes them towards the centre of the channel. The parabolic nature of the fluid velocity profile directs the shear gradient induced lift force $F_{L S}$ towards the channel wall, acting against the $F_{L W}$. The net inertial force $F_{L}$ [the dashed curve in Figure 3(a)] exhibits two dynamic stable equilibrium positions located about 0.2 times the height of the channel away from the walls. In addition, these equilibrium positions move closer to channel walls with increasing Reynolds number. ${ }^{25}$

The n-DEP force is always upwards (positive $z$ direction), shown as solid curves in Figure 3(a), which is consistent with the direction of $F_{L}$ near the bottom of the channel. The particles in this region $(z<0.2 H)$ experience a large upward net force and migrate rapidly away from the surface of the channel. If the particles reach the region where $F_{L}$ is negative, three different situations occur: (i) At low voltage, the DEP force curve intersects with the negative $F_{L}$ mirroring curve (blue dashed line) at points $\mathrm{A}$ and $\mathrm{B}$ on the bottom half of the channel, as well as at point $\mathrm{D}$ which is very close to the original inertial equilibrium position $F_{L}=0$ on the top half, Figure $3(\mathrm{~b})$. Between $\mathrm{A}$ and $\mathrm{B}\left(z_{A}<z<z_{B}\right)$, the net force of $F_{L}$ and $F_{D E P}$ is negative and is directed downwards. However, outside the A-B section $\left(z<z_{A}\right.$ or $\left.z>z_{B}\right)$, the net force is positive. Therefore, point $\mathrm{A}$ is a stable equilibrium position because the net force is directed at point $\mathrm{A}$ on both sides, which can overcome any small disturbance and draw particles back to point A. Meanwhile, point B is an unstable position because the net force on each side is directed outwards, so even a little disturbance can easily induce particles to move away. (ii) At a critical voltage $V_{c p p}$, the $F_{D E P}$ curve is tangent with the $F_{L}$ mirroring curve at a point C, Figure 3(c). This is a half stable position because the net force always points to the positive $z$ direction whether it's below or above point C. If particles were shifted below $\left(z<z_{C}\right)$, the net force will attract particles back to position C. If particles were shifted above $\left(z>z_{C}\right)$, the net force is directed upwards and the particles will never return. So very few particles are stable at point C. (iii) When the applied voltage is larger than $V_{c p p}$, there is only one node D on the top half of the channel [Figure 3(d)], and it is common to the other two situations. This point is very close to the original inertial equilibrium positions because the DEP force decreases exponentially with increasing $z$ and is negligible on the top half of the channel with almost no effect on the inertial equilibrium position.

We can conclude from this discussion that particles will gradually be levitated as the applied voltages (DEP force) are increased. 
Finally, all the particles will be focused into the equilibrium position $\mathrm{D}$ on the top half channel if the DEP force at point $\mathrm{C}$ can overcome the inertial lift force. It should be noted that the force of gravity $\left[F_{G}=(4 / 3) \pi r^{3}\left(\rho_{p}-\rho_{f}\right) g\right]$ was omitted in this analysis because the density of particles $\left(\rho_{p}=1050 \mathrm{~kg} \cdot \mathrm{m}^{-3}\right)$ was very close to its suspension medium $\left(\rho_{f}=1000 \mathrm{~kg} \cdot \mathrm{m}^{-3}\right)$.

Because of the no-slip boundary condition, the fluid velocity is zero at the walls of the channel and at its maximum velocity in the centre of the channel. The secondary flow drag $\left[F_{D}=3 \pi \mu a\left(v_{f r}-v_{p r}\right)\right]$ is enhanced if the particles are levitated towards the vertical centre of the channel by DEP force. Then, the particle trajectory along the horizontal plane will be modified. Here, $v_{f r}$ and $v_{p r}$ are the radial velocity of fluid and particles respectively. ${ }^{23}$ Finally, by using DEP force to control the vertical position of particles, their inertial focusing pattern in $3 \mathrm{D}$ can be modified and adjusted.

In order to test the above hypothesis, we developed a numerical model of the serpentine micro-channel, and released micro-particles from different heights at the inlet using particle tracking module in COMSOL Multiphysics. The results showed that particles released near the channel vertical centre can migrate and focus much faster to the channel lateral centre than those released near channel walls (Figure 4), which agreed with our hypothesis. It should be noted that in this simulation, inertial lift force were not taken into account, since there is not an explicit equation to express it, furthermore it is not easy to implement it in a curved channel with alternating curvatures.

In the following sections, the pure inertial focusing pattern and its equilibrium positions in a serpentine channel were first determined experimentally, without using the DEP force. Under different $R_{C}$, three basic focusing patterns emerged. Next, a vertical DEP force generated by IDEs was applied onto these three inertial focusing patterns respectively, to explore its effects on inertial focusing patterns. Finally, a $V_{p p} \sim R_{C}$ map was summarised to illustrate the dominance of force under different working conditions. The potential and outlook of proposed DEP-inertial microfluidics is also suggested briefly.

\section{Results and Discussions}

\section{A. Inertial focusing patterns in a serpentine channel under varying RC}

In a previous work, we discussed the mechanism of inertial focusing in a serpentine channel, ${ }^{23}$ and showed how particles with different sizes are separated according to their differential lateral positions. ${ }^{22}$ However, the inertial equilibrium positions in a vertical direction have not been shown experimentally. In order to observe the side-view, a PDMS device was fabricated from three PDMS slabs using the method reported by Guan et al. ${ }^{26}$, Figure 5(c). The PDMS device could be placed vertically onto the microscope stage, giving a clear, side view of the serpentine channel, Figure 5(b). The top view and side view enable the dynamic equilibrium positions of the particles to be determined easily.

From the top view [Left in Figure 5(d-h)], focusing streaks could be seen on the two sides of the channel at a relatively low $R_{C}$ (Figure $5 \mathrm{~d} \sim \mathrm{f}$ ), but as the $R_{C}$ increased, these two-sided focusing streaks migrated towards the centre of the channel, moved closer and closer together and then merged into a single wide streak, Figure $5(\mathrm{~g})$. This was because the secondary flow drag increased much faster than the inertial lift force within the horizontal plane as $R_{C}$ increased. They gradually dominated the two-side-directed inertial lift forces and finally pinched the particles into the centre of channel, where the net drag forces are weaker and partly cancel. As $R_{C}$ continued to increase, the focusing streak became tighter until its width could reach the single diameter of a particle. The side view (Middle in Figure $5 \mathrm{~d} \sim \mathrm{h}$ ), shows two focusing streaks near the top and bottom walls, which migrated toward the top and bottom walls as $R_{C}$ increased, in agreement with previous reports. ${ }^{19,}{ }^{20,}{ }^{25}$ The dynamic equilibrium positions within the cross section "A-a" were deduced and were illustrated schematically (green dots) in the right of Figure 5(d-h).

In summary, as $R_{C}$ increased, the dynamic equilibrium positions in a serpentine channel shifted towards the centre of channel in the lateral direction $(y)$, as well as moving closer to the walls of channel in the vertical direction $(z)$.

\section{B. Influence of n-DEP on two-sided inertial focusing pattern: modification of focusing pattern by levitation of particles}

At a relatively low $R_{C}$ (41.7 to 83.3 ), the focusing streaks at the bottom half of the channel (blue dots) were symmetric with those on the top half (green dots) along the vertical centreline, which is observed as two streaks from a top view, Figure 6(a). However, when a DEP force was applied to deflect the particles upwards, this symmetry was broken. The bottom two focusing streaks detached from the original overlapping streaks, Figure $6(\mathrm{~b}, \mathrm{c})$. A very intriguing phenomenon occurred when the two focusing streaks at the bottom migrated towards the lateral centreline of the channel as the voltage increased, and then merged to become a single streak. This means that not only by controlling the flow conditions, $R_{C}$, but also by adjusting the DEP force, inertial focusing position and pattern can be modified. The underlying mechanism for this phenomenon is that with an increased DEP force, the particles levitated upwards where the secondary flow velocity were much stronger, and the symmetrically oscillating secondary flow drag exerted a much stronger centredirected pinch effect to focus the particles towards the centre of the channel. After reaching a certain height, the two-sided focusing streaks could even be combined as a single streak at the lateral centre of channel.

When the applied voltage was higher than a corresponding critical voltage $V_{c p p}$, the single focused streak collapsed because there was no stable position in the bottom half of the channel and all the particles escaped into the top two equilibrium positions, where the DEP force was too small to have an effect. The overall trajectory of the equilibrium positions with increasing DEP force is shown as a dashed line in Figure 6(e). Since biological cells exhibit varying induced boundary polarisations dependent on applied electric frequency and their physiological states ${ }^{27}$, after choosing a proper frequency and suspending fluid, cells/particles can be separated by the differences in their dielectric properties, such as separation of viable and non-viable cells ${ }^{28,29}$, blood cells and cancer cells ${ }^{15,30}$, and CD34+ cells from peripheral-stem-cell harvests ${ }^{31}$ etc. For the inertial-DEP microfluidic device, a separation scheme could be imagined here. All the 
particles could be first squeezed down to the bottom half of the channel by a sheath flow after entering the microchannel, and then applying and adjusting a proper AC signal on the IDEs, the target particles experiencing a negative DEP could be levitated upwards and focused at the lateral centre, while particles with different dielectric properties experiencing a weak DEP force or even no DEP force, could still occupy the two-sided equilibrium positions. In this way, target particles could be isolated according to their dielectric properties, as well as particle size in our previous report. ${ }^{22}$ Therefore, modification of inertial focusing patterns by DEP force implies a more versatile capacity to differentiate and separate particles/cells by dielectric properties as well as particle size in a high-throughput tuneable manner.

\section{Influence of n-DEP on inertial single-stream focusing pattern: sheath-less three-dimensional focusing}

At a high $R_{C}(>110)$, without the existence of a DEP force, the centre-directed pinch effect from secondary flow was already strong enough to focus particles along the channel centre as a single focusing streak in the horizontal plane. In the vertical direction, particles were focused symmetrically along the top and bottom walls due to the inertial lift force, Figure 7(a). Due to the symmetry, only one focusing streak could be observed from the top view in this case. However, when a DEP force was applied in the vertical direction, the previously unobserved focusing streak from the bottom half of the channel appeared, Figures 7(b, c). The particles continued to behave as a single focusing streak, and their lateral position became more prone to follow the secondary flow streamline due to the larger secondary flow drag. The electrical voltage was gradually increased until it exceeded the critical value $V_{c p p}$, at which point the upward DEP force became strong enough to overcome the downward inertial lift force. Finally, all particles within bottom half transitioned to the equilibrium position $\mathrm{D}$ at the top of the channel, forming a truly single focusing streak in three dimensions, Figure 7(d). Such highthroughput sheath-less three-dimensional particles focusing promises wide potential applications, such as on-chip flow cytometry ${ }^{32-34}$, deformability cytometry ${ }^{35-37}$, and imaging cytometry ${ }^{38}$.

\section{Influence of n-DEP on inertial transitional focusing patterns: pinching of widely distributed particles into a tight focusing streak}

Without DEP, when Reynolds number $R_{C}$ was between 90 and 110, two-sided focusing streaks were combined as a single wide focusing streak in this flow region, which was considered as a transition between a two-sided focusing pattern and a single central focusing pattern. The particles in this pattern were distributed within a wide band near the centre of the channel, Figure 8(a). After a vertical DEP force was introduced, and within our expectations, the widely distributed particles at the bottom half of the channel migrated together if they were levitated upwards. However, it was unexpected that particles on the top half were also pinched closer in these experiments, Figure 8(c-e). The DEP force on the particles at the top half of the channel should be very small, since it decreases exponentially with the distance $z$, as discussed previously, Figure 2(c). From our understanding, the centre-directed inertial effect (secondary flow drag) and the two-side-directed inertial lift force were in a delicate balance within this transitional region, so a tiny DEP force could probably break this balance, and inadvertently promoted the central focusing. A single focusing streak in 3D could also be achieved when the electric voltage surpassed the corresponding critical voltage $V_{c p p}$. Using the DEP force to transform the transitional focusing pattern into a single central focusing pattern actually improved the inertial focusing performance and expanded the available working region of the original single central focusing. This result could be beneficial for complete separation of particles according to their differential lateral equilibrium positions in the serpentine channel, because a narrower focusing streak facilitates easier collection, and higher collection purity and efficiency.

\section{E. $V_{p p}-R_{C}$ operation map}

The critical voltage $V_{c p p}$ is a very important parameter for determining the vertical position of the particles because if the applied voltage is larger than $V_{c p p}$, the particles will be pushed over to the top half of the channel where inertial lift becomes more dominant, otherwise they will remain within the bottom half where their exact position depends on the balance of vertical inertial lift force and DEP force. A dimensionless factor $\delta$ was defined to scale with the ratio between DEP force and inertial lift force at point C (see Fig. 3).

$$
=\left|\frac{F_{D E P}}{F_{L}}\right|_{C}=\left.\frac{D_{h m}^{2} \operatorname{Re}[K(\quad)] \nabla\left|E_{r m s}\right|^{2}}{4\left|f_{L}\left(R_{C}, z\right)\right| a_{f} U_{m}^{2}}\right|_{C}
$$

From the definition, $V_{c p p}$ is the applied voltage when $\delta=1$, but if $\delta>1$ all the particles will be pushed up to top half of the channel, and when $\delta<1$, particles below the top half will remain in this region. It is difficult to determine the exact position of the tangent point $\mathrm{C}$ because the lift coefficient curve varies with channel Reynolds number $R_{C}$. Therefore, it is not practical to derive an explicit analytical expression to calculate $V_{c p p}$. However, from an experimental perspective, we could roughly determine the $V_{c p p}$ region. In the experiments, fewer particles travelled along the bottom half of the channel with increasing DEP force, as indicated by the decreased fluorescence intensity. More particles moved into equilibrium positions D in the top half of the channel leading to an increase in fluorescence intensity, Figure 9(a). Point $\mathrm{C}$ is a half stable position [Figure 3(c)], and the last position where a few particles remained at the bottom half of the channel. Therefore, the range of $V_{c p p}$ can be estimated between the maximum voltage where the bottom particles still existed and the minimum voltage where all the bottom particles disappeared shown as a dashed line with error bars in Figure 9(b). From the $V_{p p^{-}}$ $R_{C}$ map, the critical voltage $V_{c p p}$ was roughly proportional to $R_{C}$ because the order of applied voltage in DEP force was the same as $R_{C}$ $\left(\propto U_{m}\right)$ in the inertial lift force (Equ.5). Of course, the dependency of the lift coefficient $f_{L}\left(R_{C}, z\right)$ on $R_{C}$ would bring a little nonlinear nature into the $V_{c p p}-R_{C}$ relationship. Above the $V_{c p p}-R_{C}$ curve, the DEP force was strong enough to push all particles into the top half of 
the channel. Below this curve, the particles were levitated but still remain within the bottom half.

\title{
Conclusions and outlook
}

This work, for the first time, proposed a novel DEP-inertial microfluidic device that fully couples the DEP force and inertial lift force in a serpentine microchannel. Rather than using a horizontal DEP force, a vertical DEP force was used to adjust the particle equilibrium positions in the vertical direction. Due to the much stronger secondary flow drag imposed on particles when they were near the vertical centre of the channel, focusing patterns and positions of particles along horizontal plane could be adjusted by their vertical position. The mechanism of this DEP-inertial microfluidic device was also investigated numerically and experimentally. The potential advantages of DEP-inertial microfluidic device includes: (i) Real-time controllability; (ii) Three dimensional single stream focusing; (iii) Enhanced focusing quality, and the ability to manipulate particles; (iv) The DEP force could be used as a force probe to investigate the mechanism of particle inertial focusing process; (v) Surface-patterned microelectrodes could also possibly work as an impedance-based detector, to simultaneously enumerate and differentiate the passing micro-particles; (vi) Separation of particles/cells by size as well as by dielectric property; (vii) Utilisation of different focusing patterns of particles by varying the DEP forces in real-time to act as an online active sorter. In summary, this DEP-inertial device offers the possibility of combining a high-throughput manner with accurate real-time controllability, which is expected to provide a versatile and robust feedback-controlled platform for manipulating and separating particles and cells.

\section{Acknowledgement}

This work was partially supported by the University of Wollongong through a UIC grant and China Scholarship Council.

\author{
Notes \\ ${ }^{a}$ School of Mechanical, Materials and Mechatronic Engineering, University of Wollongong, Wollongong, NSW 2522, Australia. Fax: +61 242213238 ; \\ Tel: +61 24221 3490; E-mail: weihuali@uow.edu.au \\ ${ }^{b}$ Queensland Micro- and Nanotechnology Centre, Griffith University, Brisbane QLD 4111, Australia. Fax: +61 07 373 58021; Tel: +61 (07) 373 53921; \\ E-mail:nam-trung.nguyen@griffith.edu.au \\ ${ }^{c}$ Department of Bioengineering, University of California, 420 Westwood Plaza, 5121 Engineering V, P.O. Box 951600, Los Angeles, CA, 90095, USA. E- \\ mail: dicarlo@seas.ucla.edu; Fax: +1 310794 5956; Tel: +1 3109833235 \\ ${ }^{d}$ California NanoSystems Institute, 570 Westwood Plaza, Building 114, Los Angeles, CA, 90095, USA
}

\section{References}

1 J. Kim, M. Johnson, P. Hill and B. K. Gale, Integr. Biol., 2009, 1, 574-586.

2 T. H. Schulte, R. L. Bardell and B. H. Weigl, Clin. Chim. Acta, 2002, 321, 1-10.

3 M. A. Shannon, P. W. Bohn, M. Elimelech, J. G. Georgiadis, B. J. Marinas and A. M. Mayes, Nature, 2008, 452, 301-310.

4 P. S. Dittrich and A. Manz, Nat. Rev. Drug Discovery, 2006, 5, 210-218.

5 J. I. Park, A. Saffari, S. Kumar, A. Günther and E. Kumacheva, Annu. Rev. Mater. Res., 2010, 40, 415-443.

6 B. Çetin and D. Li, Electrophoresis, 2011, 32, 2410-2427.

7 T. P. Forbes and S. P. Forry, Lab Chip, 2012, 12, 1471-1479.

8 Z. Wang and J. Zhe, Lab Chip, 2011, 11, 1280-1285.

9 M. Yamada, M. Nakashima and M. Seki, Anal. Chem., 2004, 76, 5465-5471.

10 L. R. Huang, E. C. Cox, R. H. Austin and J. C. Sturm, Science, 2004, 304, 987-990.

11 D. Di Carlo, Lab Chip, 2009, 9, 3038-3046.

12 S. Yan, J. Zhang, M. Li, G. Alici, H. Du, R. Sluyter and W. Li, Sci. Rep., 2014, 4, Article number 5060.

13 E. Ozkumur, A. M. Shah, J. C. Ciciliano, B. L. Emmink, D. T. Miyamoto, E. Brachtel, M. Yu, P.-i. Chen, B. Morgan and J. Trautwein, Sci. Transl. Med., 2013, 5, 179ra47.

14 N. M. Karabacak, P. S. Spuhler, F. Fachin, E. J. Lim, V. Pai, E. Ozkumur, J. M. Martel, N. Kojic, K. Smith and P.-i. Chen, Nat. Protoc., 2014, 9, 694710.

15 H. S. Moon, K. Kwon, S. I. Kim, H. Han, J. Sohn, S. Lee and H. I. Jung, Lab Chip, 2011, 11, 1118-1125.

16 D. C. Duffy, J. C. McDonald, O. J. A. Schueller and G. M. Whitesides, Anal. Chem., 1998, 70, 4974-4984.

17 M. Li, S. Li, J. Wu, W. Wen, W. Li and G. Alici, Microfluid. Nanofluid., 2012, 12, 751-760

18 S. H. Baek, W.-J. Chang, J.-Y. Baek, D. S. Yoon, R. Bashir and S. W. Lee, Anal. Chem., 2009, 81, 7737-7742.

19 E. S. ASMOLOV, J. Fluid Mech., 1999, 381, 63-87.

20 D. Di Carlo, D. Irimia, R. G. Tompkins and M. Toner, Proc. Natl. Acad. Sci. U.S.A., 2007, 104, 18892-18897.

21 J. Zhang, S. Yan, W. Li, G. Alici and N.-T. Nguyen, RSC Advances, 2014, 4, 33149-33159.

22 J. Zhang, S. Yan, R. Sluyter, W. Li, G. Alici and N.-T. Nguyen, Sci. Rep., 2014, 4, Article number 4527.

23 J. Zhang, W. Li, M. Li, G. Alici and N.-T. Nguyen, Microfluid. Nanofluid., 2013, 17, 305-316.

24 D. J. Collins, T. Alan and A. Neild, Lab Chip, 2014, 14, 1595-1603.

25 J.-P. MATAS, J. F. MORRIS and É. GUAZZELLI, J. Fluid Mech., 2009, 621, 59-67.

26 G. Guan, L. Wu, A. A. Bhagat, Z. Li, P. C. Chen, S. Chao, C. J. Ong and J. Han, Sci. Rep., 2013, 3, Article number 1475.

27 H. Li and R. Bashir, Sensors and Actuators B: Chemical, 2002, 86, 215-221.

28 B. H. Lapizco-Encinas, B. A. Simmons, E. B. Cummings and Y. Fintschenko, Anal. Chem., 2004, 76, 1571-1579.

29 H. Shafiee, M. B. Sano, E. A. Henslee, J. L. Caldwell and R. V. Davalos, Lab Chip, 2010, 10, 438-445.

30 A. Alazzam, I. Stiharu, R. Bhat and A. N. Meguerditchian, Electrophoresis, 2011, 32, 1327-1336.

31 M. Stephens, M. Talary, R. Pethig, A. Burnett and K. Mills, Bone Marrow. Transplant., 1996, 18, 777-782. 
32 J. Oakey, R. W. Applegate Jr, E. Arellano, D. D. Carlo, S. W. Graves and M. Toner, Anal. Chem., 2010, 82, 3862-3867.

33 K. C. Cheung, M. Di Berardino, G. Schade-Kampmann, M. Hebeisen, A. Pierzchalski, J. Bocsi, A. Mittag and A. Tárnok, Cytometry A, 2010, 77, 648-666.

34 D. C. Spencer, G. Elliott and H. Morgan, Lab Chip, 2014, 14, 3064-3073.

35 T. Henry, D. R. Gossett, Y. S. Moon, M. Masaeli, M. Sohsman, Y. Ying, K. Mislick, R. P. Adams, J. Rao and D. Di Carlo, Sci. Transl. Med., 2013, 5, $212 \mathrm{ra} 163$.

36 J. S. Dudani, D. R. Gossett, T. Henry and D. Di Carlo, Lab Chip, 2013, 13, 3728-3734.

37 D. R. Gossett, T. Henry, S. A. Lee, Y. Ying, A. G. Lindgren, O. O. Yang, J. Rao, A. T. Clark and D. Di Carlo, Proc. Natl. Acad. Sci. U.S.A., 2012, 109, 7630-7635.

38 K. Goda, A. Ayazi, D. R. Gossett, J. Sadasivam, C. K. Lonappan, E. Sollier, A. M. Fard, S. C. Hur, J. Adam and C. Murray, Proc. Natl. Acad. Sci. U.S.A., 2012, 109, 11630-11635.

39 J.-P. MATAS, J. F. MORRIS, GUAZZELLI, Eacute and LISABETH, J. Fluid Mech., 2004, 515, 171-195. 

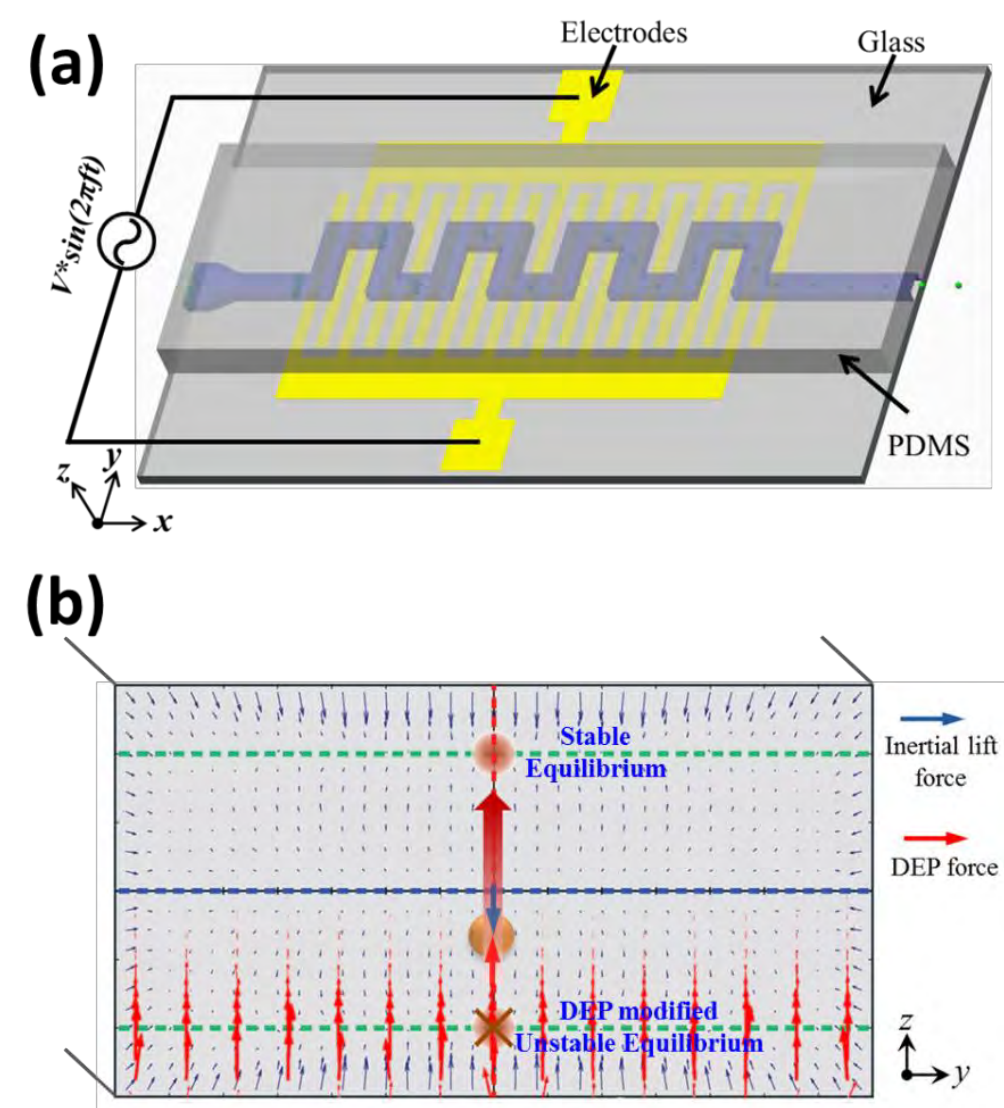

Figure 1 (a) Schematic of DEP-inertial microfluidic device. (b) Distribution of inertial lift force vectors and DEP force vectors within a cross section of DEP-inertial microfluidic device. The serpentine micro-channel is embedded in the PDMS slab, and the slab is bonded with a clean glass slide where interdigitated electrodes (IDEs) are patterned. The particle suspension is delivered into the micro-channel by a syringe pump. An alternating current (AC) signal is imported into the IDEs, activating DEP force to levitate particles along the vertical $z$ direction. DEP force competes with inertial lift force along $z$ direction, thus alters the final vertical position of particles. As a result, the inertial focusing process and pattern can be tuned in three dimensions. 

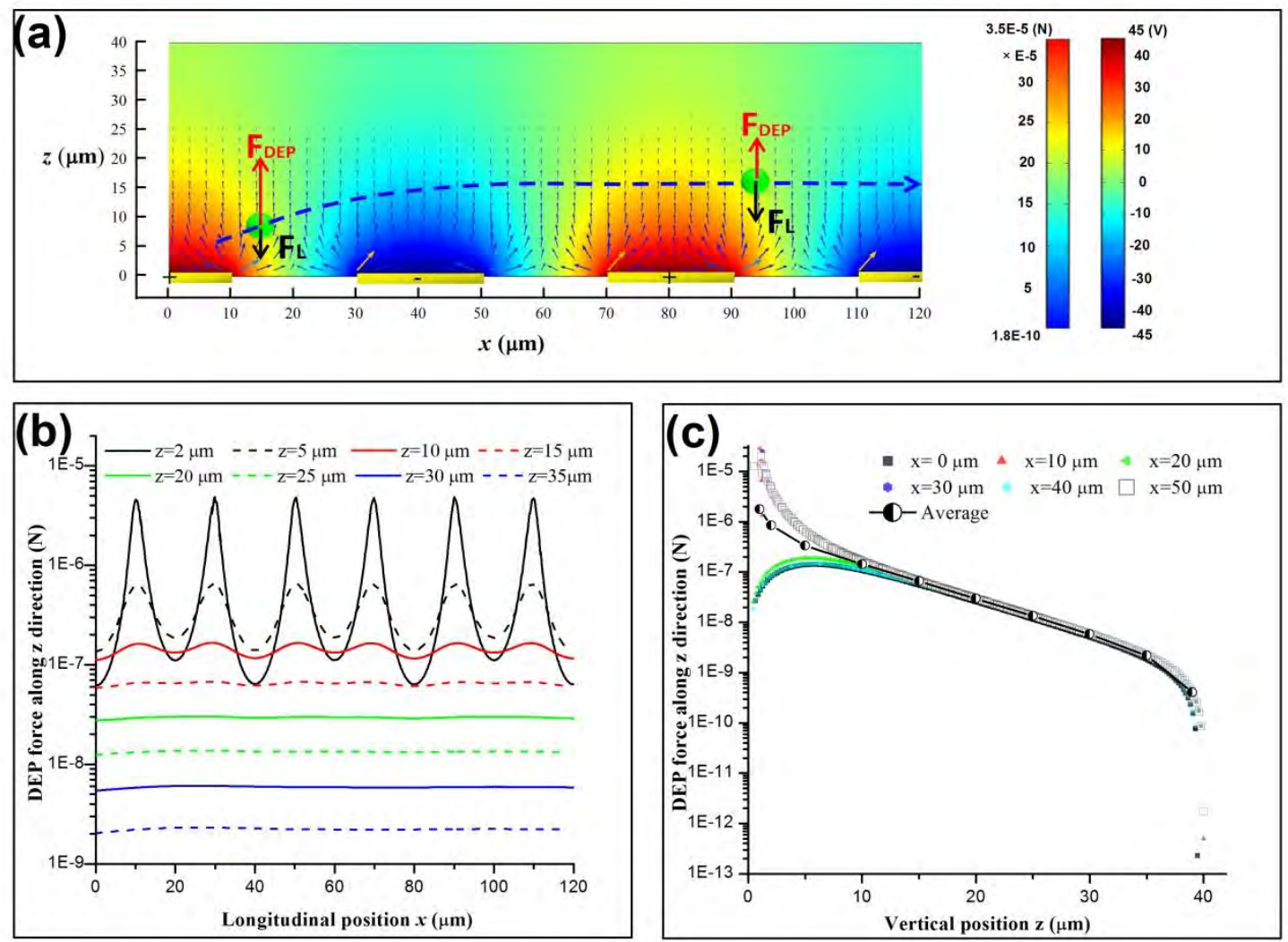

Figure 2 (a) The electrical field and n-DEP force distribution within a longitudinal section. The arrow vectors represent the magnitude and direction of nDEP force. The magnitude of n-DEP force along $\mathrm{z}$ direction is implied by the vectors' colour, and corresponds to the left colour bar. Colour map surface shows the electric field distribution, and corresponds to the right colour bar. (b) Longitudinal distribution of n-DEP force along $z$ direction $\left(F_{D E P_{z}}\right)$. $(\mathrm{c})$ Vertical distribution of $\mathrm{n}$-DEP force along $\mathrm{z}$ direction $\left(F_{D E P_{z}}\right)$. COMSOL Multi-physics 4.3 was used to calculate the electric field, and the DEP force was calculated using equation (3). Particle diameter is $10 \mu \mathrm{m}$, and $\operatorname{Re}[K(\omega)]$ for polystyrene beads in DI water at electric frequency of $1 \mathrm{MHz}$ is approximated as -0.5 . 

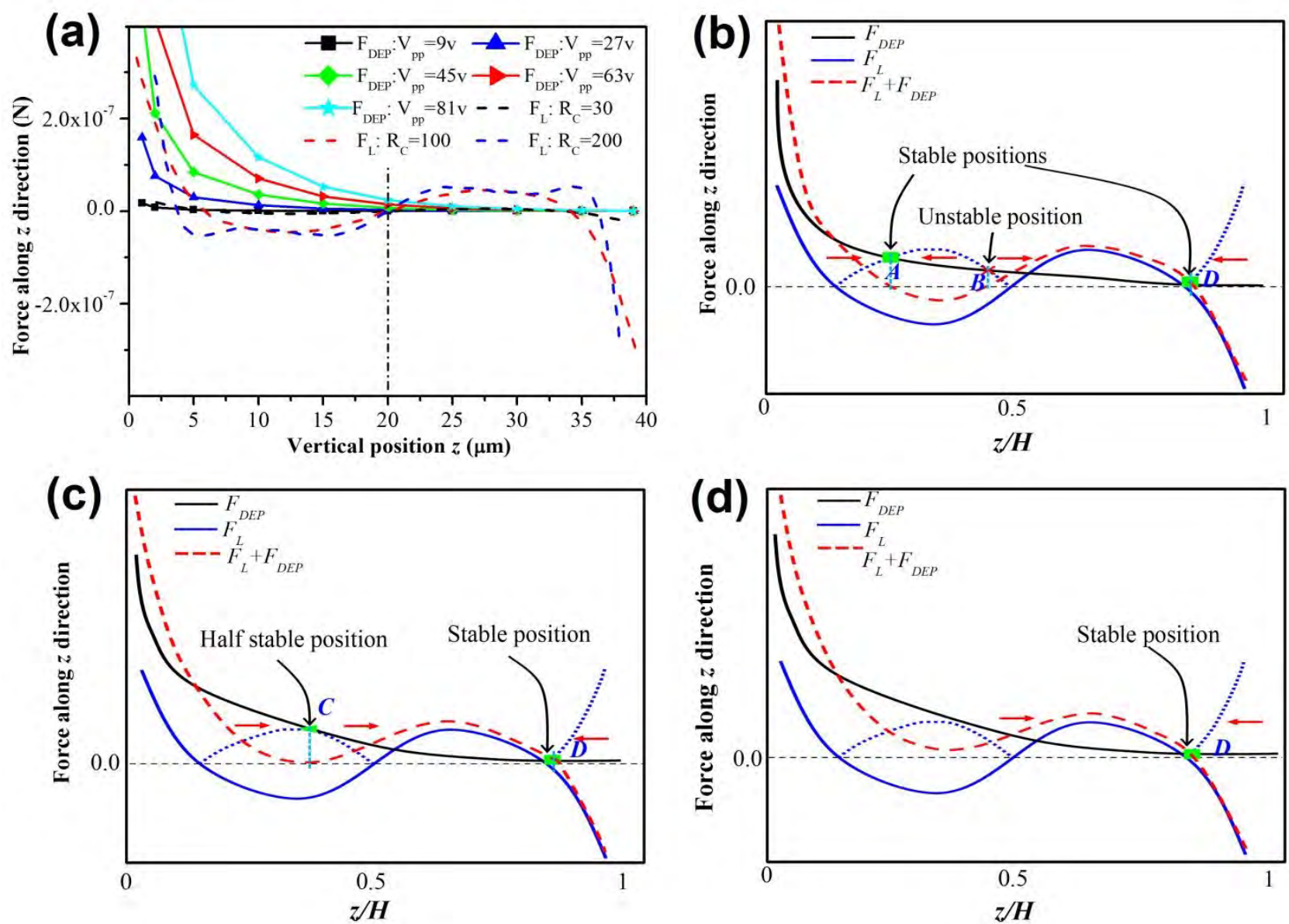

Figure 3 (a) The distribution of dielectrophoresis (DEP) force $F_{D E P}$ and inertial lift force $F_{L}$ along the height of the channel $z$. The inertial lift force is obtained from the analytical solution from MATAS et al. ${ }^{25,39}$ and n-DEP force is obtained from the simulation results by COMSOL Multi-physics. Particle diameter is $10 \mu \mathrm{m}$. (b) At relative low applied voltages, two stable equilibrium positions (A and D) along $z$ exist, which are modifications of the original inertial equilibrium positions. The direction of the net force (red arrows) on both sides of stable positions will always point to themselves because they can overcome any small disturbance. However, point B is in an unstable position because the net force along both sides is directed outwards, and a little disturbance will induce the particles to move away from point B. The blue dashed curve is the mirror image of the $F_{L}$ negative part on a positive coordinate. (c) At a critical voltage $\left(V_{c p p}\right)$, the $F_{D E P}$ curve is tangential with the $F_{L}$ negative mirroring curve at point $\mathrm{C}$, which is in a half stable (saddle) position. If particles were shifted to below $\left(z<z_{C}\right)$, the net force will attract particles back to position $\mathrm{C}$, but when the particles were shifted to above $(z>$ $z_{C}$ ), the net force is directed upwards and particles will never return. (d) When the applied voltage is higher than the critical voltage $V_{c p p}$, there is only one vertical equilibrium position $\mathrm{D}$ on the upper half of the channel. 


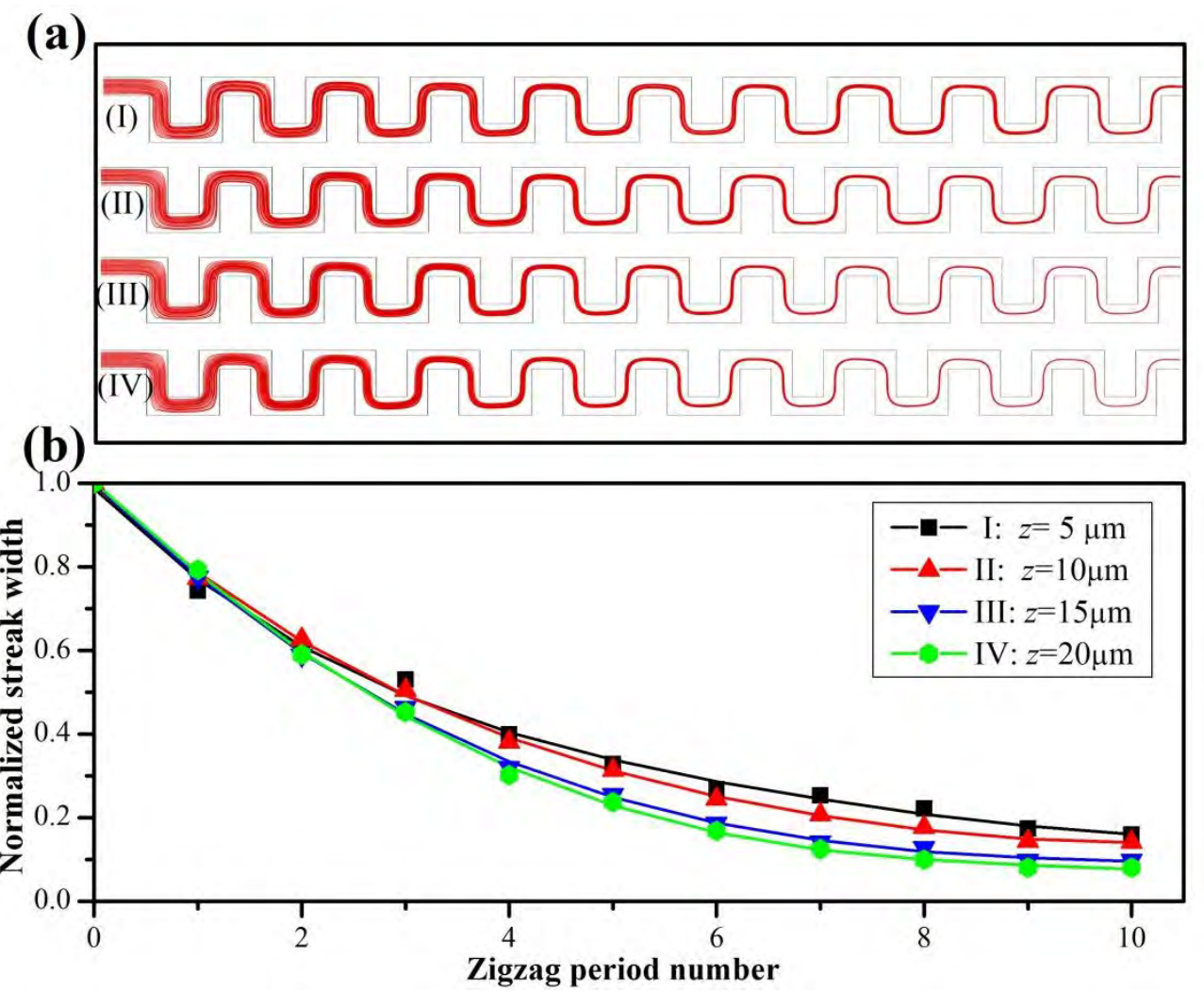

Figure 4 (a) Simulated particles' trajectories released from different heights. (b) Normalized particle streak widths after traveling through different channel lengths. The particles released near the channel vertical centre focus into the channel lateral centre much faster than that release near channel walls, because fluid velocity is at maximum at channel centre, and its corresponding secondary flow drag and centrifugal force is much stronger than that near channel walls. 


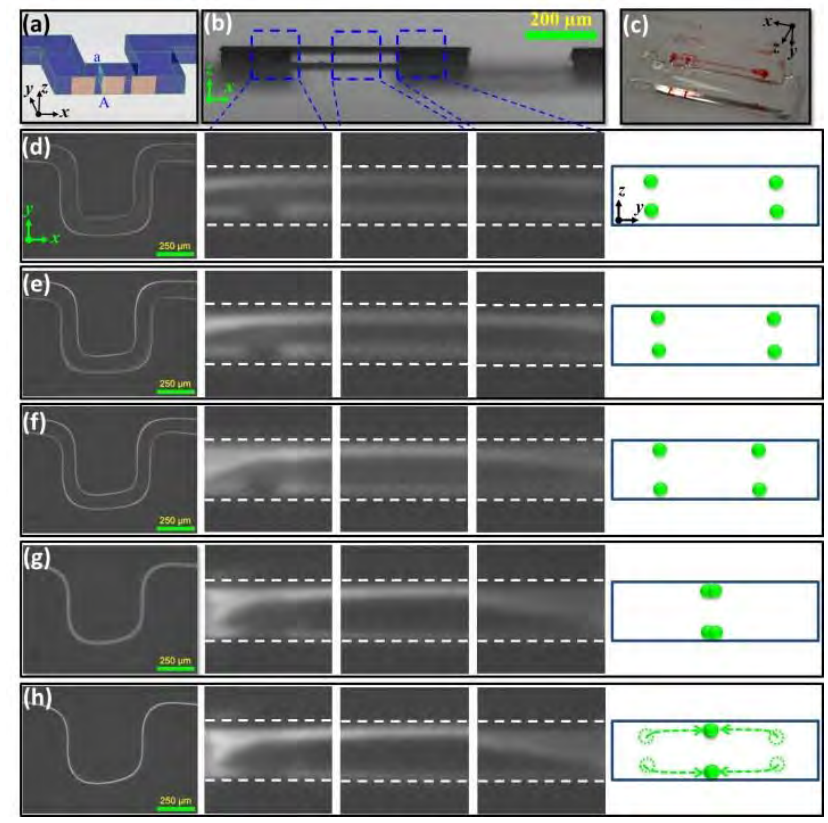

Figure 5 (a) Schematic 3D structure of one period in the serpentine channel and (b) its side view optical image; (c) The fabricated PDMS device was used to observe the serpentine channel from both top-down and side-side view in the experiments. (d-h) Inertial particle focusing patterns under varying flow conditions: (d) $R_{C}=41.7$; (e) $R_{C}=62.5$; (f) $R_{C}=83.3$; (g) $R_{C}=104.2$; (h) $R_{C}=125$. (Left) Top views of fluorescent particles trajectory at the last zigzag period of serpentine; (Middle) Sided views of fluorescent particles at the last zigzag period; and (Right) The dynamic equilibrium positions of particles at crosssection A-a. 

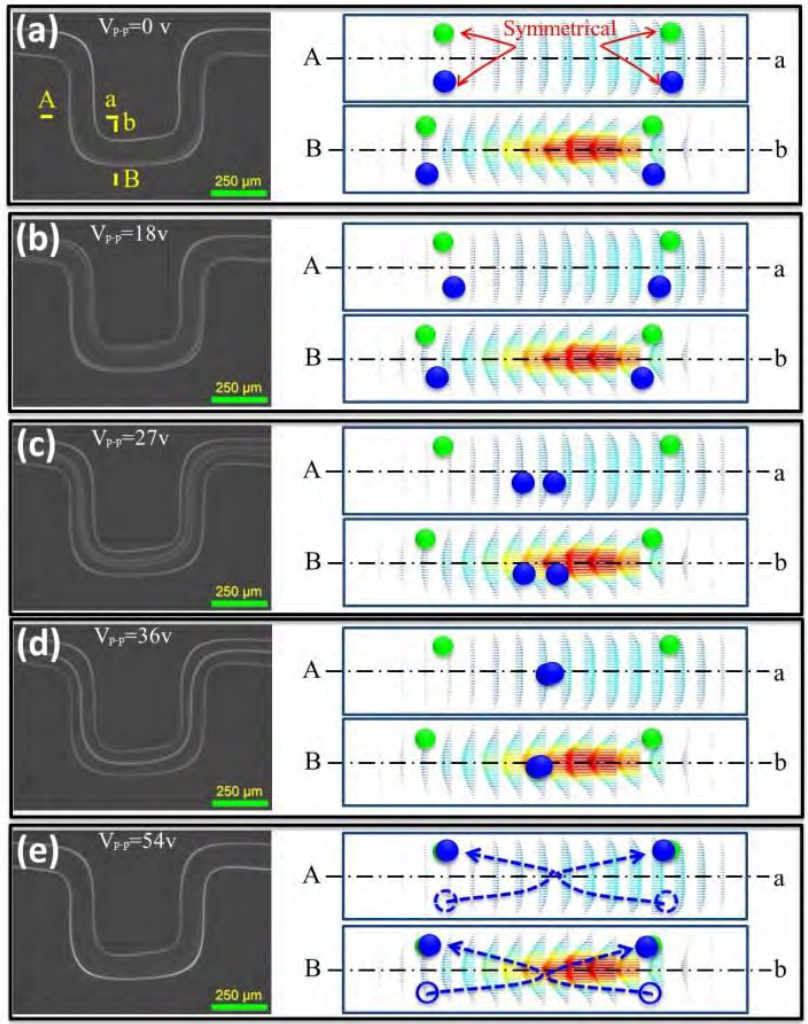

Figure 6 Migration of particle focusing patterns under different AC voltages of (a) $V_{p-p}=0 \mathrm{v}$, (b) $V_{p-p}=18 \mathrm{v}$, (c) $V_{p-p}=27 \mathrm{v}$, (d) $V_{p-p}=36 \mathrm{v}$ and (e) $V_{p-p}=54 \mathrm{v}$. (Left) trajectory of fluorescent particles from Top view, and (Right) their corresponding postulated dynamic equilibrium positions within two typical cross-sections "A-a" and "B-b". Flow condition is $R_{C}=62.5$. 


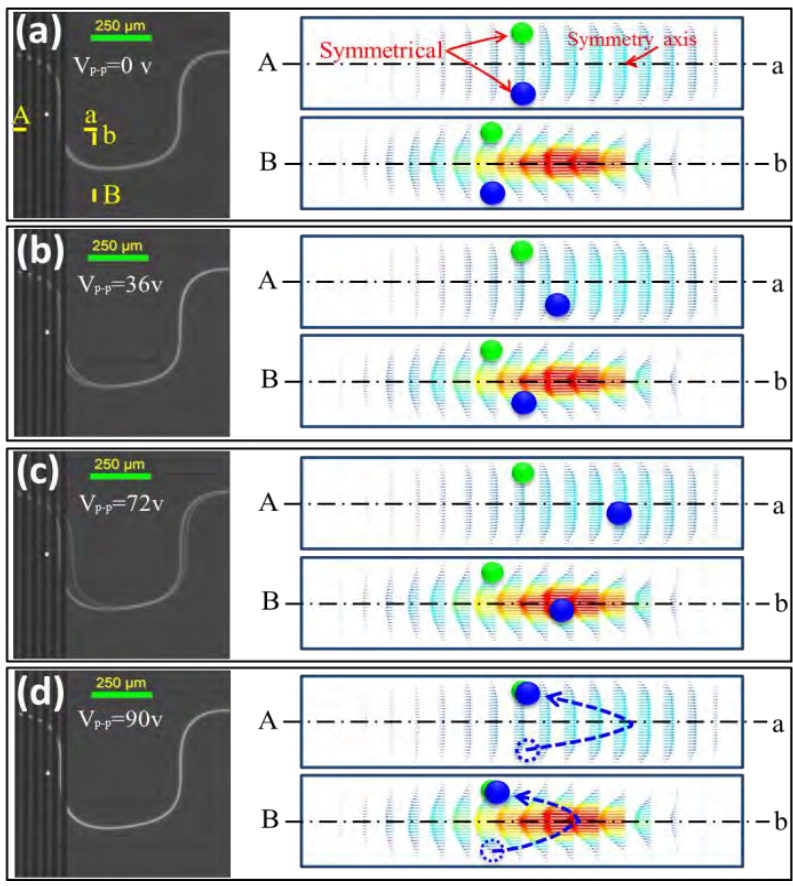

Figure 7 Migration of particle single central focusing patterns under different AC voltages of (a) $V_{p-p}=0 \mathrm{v}$, (b) $V_{p-p}=36 \mathrm{v}$, (c) $V_{p-p}=72 \mathrm{v}$ and (d) $V_{p-p}=90 \mathrm{v}$. (Left) trajectory of fluorescent particles from top view, and (Right) their corresponding postulated dynamic equilibrium positions within two typical crosssections "A-a" and "B-b". Flow condition is $R_{C}=114.6$. 


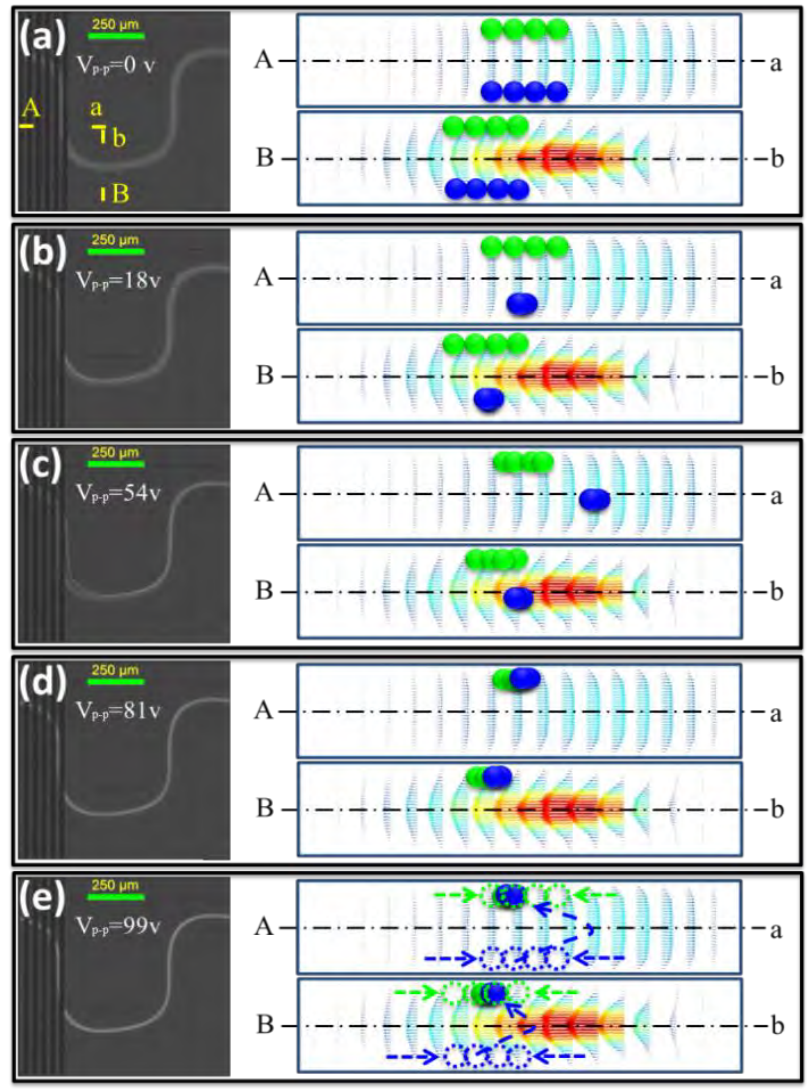

Figure 8 Migration of particle transitional focusing patterns under different AC voltages of (a) $V_{p-p}=0 \mathrm{v}$, (b) $V_{p-p}=18 \mathrm{v}$, (c) $V_{p-p}=54 \mathrm{v}$, (d) $V_{p-p}=81 \mathrm{v}$ and (e) $V_{p-p}=99 \mathrm{v}$. (Left) trajectory of fluorescent particles from top view, and (Right) their corresponding postulated dynamic equilibrium positions within two typical cross-sections "A-a" and "B-b". Flow condition is $R_{C}=99$. 

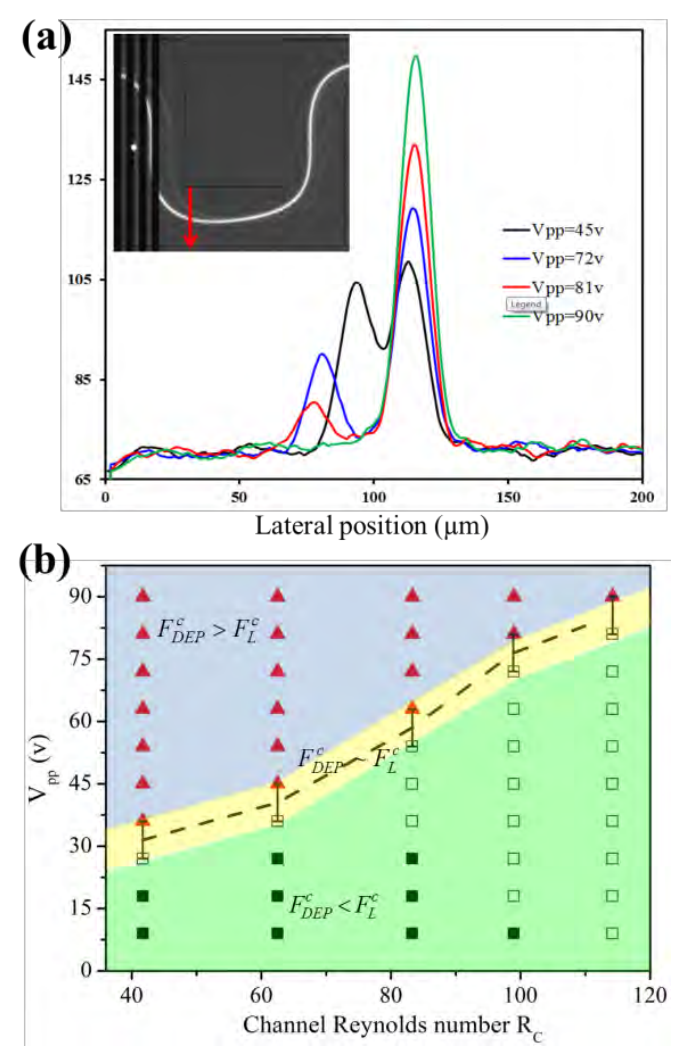

Figure 9 (a) Distribution of lateral fluorescence intensity with various applied voltages; (b) $V_{p p}-R_{C}$ map illustrates the working range of DEP-inertial microfluidic device. The red triangles are experimental data where particles are pushed onto the top half of the channel. The applied voltages are larger than the critical voltage. The empty rectangles are experimental data where particles under bottom half part of channel are focused into a single central focusing streak under the effects of DEP force. The solid rectangles are the experimental data where DEP force is not sufficient to alter particle inertial focusing pattern in bottom half part of channel. 